Commission ${ }^{15}$ with a Peacebuilding Support Office and Peacebuilding Fund offers a potentially valuable mechanism to integrate different actors and approaches. The central challenge for this new body and indeed for the States that endorsed its creation will be to move from a recognition of the complexity of the challenge to effecting change in practice. This will require an acceptance of new ways of doing business that overcome particularistic interests and resist the pull of bureaucratic inertia. Political will is therefore the most important commodity in developing the role of the Peacebuilding Commission in a meaningful way.

15 United Nations General Assembly 2005: Final document of the High-level Plenary Meeting of the General Assembly, A/60/L.1, of 15 September 2005. See also the contribution by Ulrich Schneckener and Silke Weinlich in this issue.
There is a clear need to better link, sequence and optimise the diverse component parts of post-conflict peacebuilding. This article has argued that a security governance perspective - combining emphasis on the effective and efficient provision of security with the corresponding need for good governance of the security sector - provides a useful framework to better link these different stakeholders in the security dimensions of post-conflict peacebuilding. Much can be learned across different issue areas in terms of good (and bad) practice which can be used to inform future interventions. But the value of improved coordination and cooperation must be measured on the ground where gaps between policy and practice are most acutely felt.

\title{
Das Treuhandsystem der Vereinten Nationen als Mittel zum Peacebuilding?
}

\author{
Patrick Sutter*
}

\begin{abstract}
With the end of the Cold War a more pronounced view on states' domestic responsibilities emerged, as states are today understood to be internationally liable for their domestic conducts. In parallel the humanitarian principle to safeguard populations at risk, among others, accords the international community a right to intervene into states' domestic affairs. This article argues that the right of self determination paired with the right of intervention legalizes international nation- and peacebuilding efforts under the terms of the UN charter's long-forgotten provisions on trusteeships. It argues that these provisions allow a country's inhabitants or its eventual legitimate occupying power to transfer states to trustee status and thus UN guardianship. Trusteeship law could so serve as the legal basis for effective UN peacebuilding efforts.
\end{abstract}

Keywords: Vereinte Nationen, Treuhandsystem, Peacebuilding, VN-Charta

\section{Einleitung}

$\mathrm{S}$ eit dem Ende der relativ stabilen Ordnung des Kalten Krieges sieht sich die Staatengemeinschaft immer öfter veranlasst, mittels Interventionen eskalierende oder bereits vollständig eskalierte Konflikte zu beenden. Dies hat nicht zuletzt damit zu tun, dass sich die Staatengemeinschaft selbst Verpflichtungen auferlegt hat, die sie, will sie glaubwürdig bleiben, letztendlich auch zu erfüllen hat. Im Vordergrund stehen der Schutz der Zivilbevölkerung ${ }^{1}$ und das Verbot gewisser Waffenarten ${ }^{2}$ - also zwei der drei Grundpfeiler des klas-

\footnotetext{
* Patrick Sutter, lic, iur. HSG, ist in einer Anwaltskanzlei in Schwyz (Schweiz) tätig und hat kürzlich an der Universität St. Gallen seine rechtswissenschaftliche Dissertation zum Thema »Wissenschaft und Ethik in der Rechtsetzung « eingereicht. Er ist Milizoffizier in der Schweizer Armee und dient dort als Rechtsberater in der Sektion Kriegsvölkerrecht.

1 In diesen Fällen ist von »humanitären Interventionen« die Rede, so z.B. im Kosovo.

2 Es geht dabei v.a. um ABC-Waffen. Die (nicht nur militärische) Intervention im Irak war ursprünglich damit begründet.
}

sischen humanitären Völkerrechts. Es handelt sich bei diesen Interventionspflichten insofern gleichzeitig um Interventionsrechte, als die von der Intervention betroffenen Staaten keine verbotene Einmischung in interne Angelegenheiten geltend machen können. Ob ein solches Interventionsrecht besteht, entscheidet im System der kollektiven Sicherheit allein der Sicherheitsrat (SR) der Vereinten Nationen (VN). ${ }^{3}$ Alle nachfolgenden Ausführungen stehen deshalb unter dem Vorbehalt, dass die Intervention selbst völkerrechtskonform ist. ${ }^{4}$

\footnotetext{
3 Siehe etwa Donner, Michael 1995: Die Begrenzung bewaffneter Konflikte durch das moderne jus ad bellum, in: Archiv für Völkerrecht 33, 168ff.

4 »If this is not the case, as the two most recent cases of Kosovo and Iraq demonstrate, then we immediately run into severe problems of legitimacy and credibility of the UN system. This is why attempts to dump postconflict situations requiring reconstruction in the lap of the UN following unilateral or multilateral interventions not sanctioned by the SC should be strongly resisted. The SC and the UN's collective security mechanism should not be allowed to become tools in the hands of intervening powers who have done so without UNSC authorization, as happened in Kosovo and in Iraq" (Ayoob, Mohammed 2005: Post-Conflict Reconstruction, 1, unter: http://www.un-globalsecurity.org/pdf/Ayoob_paper_postconflict.pdf). Vergleiche auch A/Res/60/1, Ziff. 77.
} 
Das Spektrum der interventionistischen Handlungsoptionen ist äußerst breit und Gegenstand intensivierter Forschung. ${ }^{5}$ Die militärische Intervention soll erst als Ultima Ratio zur Anwendung kommen. Trotzdem bedeutet das in letzter Konsequenz, dass das Recht im Krieg (ius in bello) letztlich ein Recht zum Krieg (ius ad bellum) bereitstellt. Zugespitzt kann man also sagen, dass der im Schutz der Zivilbevölkerung und dem Verbot gewisser Waffenarten zum Ausdruck kommende Siegeszug der humanitären Idee die Bereitschaft der Staatengemeinschaft impliziert, als letztes Mittel zum Krieg zu greifen. ${ }^{6}$ Dieses Paradoxon ist Ergebnis der aporetischen Situation, dass es keine ethisch erhabene Position der Nichtentscheidung mehr gibt, weil in jedem Fall gravierende negative Folgen zu verzeichnen sind; insbesondere kann sich ein absoluter Pazifismus als ethisch verwerflich erweisen. ${ }^{7}$

Kommt es unter diesen Voraussetzungen zu einer völkerrechtlich legitimen militärischen Intervention, so gelingt es aufgrund der militärischen Überlegenheit der Staatengemeinschaft meist relativ schnell, die eigentlichen Kampfhandlungen zu beenden. Doch damit ist erst der Anfang gesetzt für eine schwierige, weil äußerst komplexe Aufgabe: die nachhaltige Konsolidierung des Friedens, nachfolgend Peacebuilding genannt. Wie sogleich zu zeigen sein wird, ist es nicht der Willkür der Staatengemeinschaft überlassen, ob sie sich dieser Aufgabe annehmen will. Wenn sie sich zur Intervention entschließt, verpflichtet sie das Völkerrecht, in der Folge für Frieden und Stabilität zu sorgen, bevor sie sich zurückziehen darf.

Die bisherigen Erfahrungen - vor allem in den diesbezüglich sehr intensiven letzten 15 Jahren - zeigen, dass die Erfolge der Staatengemeinschaft relativ dünn gesät sind. Oft fielen die Staaten nach dem Abzug der internationalen Truppen wieder ins Chaos, ja teilweise verloren die internationalen Truppen sogar die Kontrolle über das Gebiet und wurden zum Abzug gezwungen. Es gilt deshalb, diese Erfahrungen auszuwerten und die entsprechenden Schlussfolgerungen für künftige Fälle zu ziehen.

Zugleich deuten die Insuffizienzen der Vergangenheit an, dass sich die Staatengemeinschaft nach weiteren Optionen umsehen muss, um die Schwierigkeiten des Peacebuilding zu meistern. Im Zuge der Reformbestrebungen der VN schlug der vom VN-Generalsekretär eingesetzte »High-level Panel on Threats, Challenges, and Change« die Schaffung einer Peacebuilding Commission (PBC) vor. ${ }^{8}$ Der Generalsekretär nahm diese Idee in seinem Bericht »In Larger Freedom« vom März

5 M.E. grundlegend Bernauer, Thomas/Ruloff, Dieter 1999: Introduction and Analytical Framework, in: Bernauer, Thomas/ Ruloff, Dieter (Hrsg.): The Politics of Positive Incentives in Arms Control, Columbia, $1 \mathrm{ff}$

6 Siehe etwa Schweizer, Beat 2004: Moral dilemmas for humanitarianism in the era of "humanitarian « military interventions, in: International Review of the Red Cross 86, $547 \mathrm{ff}$.

7 Die letzten Jahre haben gezeigt: Während die Europäer dazu tendieren, die Folgen eines Abseitsstehens zu tief zu bewerten, führt die optimistische Sichtweise der USA eher dazu, die Folgen einer Intervention zu unterschätzen. Zur Frage der Interventionskapazitäten vergleiche $O^{\prime} H a n l o n$, Michael/ Singer, P.W. 2004: The Humanitarian Transformation: Expanding Global Intervention Capacity, in: Survival 46, 77ff.

8 A More Secure World: Our shared responsibility, Report of the Secretary General's High-level Panel on Threats, Challenges, and Change, United Nations 2004, Ziff. 263, unter: http://www.un.org/secureworld/report2.pdf.
2005 auf. ${ }^{9}$ Dementsprechend beschloss die VN-Generalversammlung anlässlich ihrer 60. Tagung im September 2005 die Einrichtung einer solchen Kommission mit beratender Funktion. ${ }^{10}$ Es wird letztlich von der konkreten Ausgestaltung der PBC abhängen, ob sie die ausgemachte Lücke in der Ordnung der Vereinten Nationen zu füllen vermag.

In den Überlegungen um die Reform scheint hingegen nicht Gegenstand der Erwägungen gewesen zu sein, das mit dem Ende der Dekolonisierung brachliegende Treuhandsystem der VN einer neuen Aufgabe, eben dem Peacebuilding, zuzuführen. Die nachfolgende Untersuchung bezweckt deshalb, zu klären, ob eine allfällige, auch nach der Schaffung der PBC bestehende Lücke durch das Treuhandsystem gefüllt werden könnte. Welche rechtlichen und politischen Hindernisse stehen einer direkten oder adaptierten Anwendung des VN-Treuhandsystems für das Peacebuilding entgegen? Welche Argumente sprechen dafür? Ausgangspunkt für die Beantwortung dieser Fragen muss das völkerrechtliche Selbstbestimmungsrecht sein (Teil 2). In diesen Argumentationsrahmen kann anschließend die Problematik der Anwendung des VN-Treuhandsystems für das Peacebuilding eingebettet werden (Teil 3). Nachdem diese juristischen Erwägungen zeigen, unter welchen Voraussetzungen die Staatengemeinschaft von diesem Instrument Gebrauch machen kann, folgen abschließend Gedanken dazu, ob und, wenn ja, wann sie davon auch Gebrauch machen soll (Teil 4).

\section{Grundlagen}

\subsection{Das Selbstbestimmungsrecht des Volkes}

Jedes Staatsvolk und jedes Volk eines werdenden Staates können das völkerrechtliche Selbstbestimmungsrecht in Anspruch nehmen. Mit den »werdenden Staaten« meinte das Völkerrecht in der Zeit der Entkolonialisierung nach dem Zweiten Weltkrieg die Kolonien. ${ }^{11}$ Ein sich auf das Selbstbestimmungsrecht berufendes »Staatsvolk « hingegen ist typischerweise ein Volk, welches fremder Besetzung oder Beherrschung unterworfen ist. ${ }^{12}$ Das irakische Volk war z.B. trotz Besetzung durch die alliierten Kräfte zu jedem Zeitpunkt ein Staatsvolk, weshalb ihm jederzeit das völkerrechtliche Selbstbestimmungsrecht zustand. Dies zeigt sich in der SR-Resolution 1483 (2003), die »the right of the Iraqi people freely to determine their own political future « betonte, und verlangte, »that the day when Iraqis govern themselves must come quickly«. Dies betrifft allerdings nur die Frage, wann die Besetzung beendet werden muss; während der Besetzung richtet sich die Handlungsfreiheit der Besatzungsmacht nach dem humanitären Völkerrecht

\footnotetext{
9 In Larger Freedom: Towards Security, Development and Human Rights for All, Report of the Secretary General of the United Nations for decision by Heads of State and Government in September 2005, A/59/2005, Addendum 2 (http://www.un.org/largerfreedom/add2.htm).

10 Vgl. Beitrag von Ulrich Schneckener und Silke Weinlich in diesem Heft.

11 Für eine vertiefte Betrachtung siehe Sutter, Patrick 2003a: Nation Building: Stabilität durch Selbst- oder durch Fremdbestimmung?, in: Patrick Sutter (Hrsg.): Selbstbestimmung und Recht,), Festgabe für Rainer J. Schweizer zum 60. Geburtstag, Zürich, $208 \mathrm{ff}$.

12 Ermacora, Felix 2000: Autonomie als innere Selbstbestimmung, in: Archiv für
} Völkerrecht 38, 290. 
(insbesondere nach Art. 47 ff. des Genfer Abkommens von 1949 über den Schutz von Zivilpersonen in Kriegszeiten).

Die Staatsvölker bzw. Völker von werdenden Staaten können das äußere Selbstbestimmungsrecht gemäß der Friendly Relations Declaration vom 24. Oktober 1970 (Res. Nr. 2625/XXV) auf vier verschiedene Arten wahrnehmen:

1. Zur Gründung eines souveränen und unabhängigen Staates;

2. Zur freien Vereinigung mit einem unabhängigen Staat;

3. Zur freien Eingliederung in einen solchen Staat;

4. Zur Begründung eines anderen, durch das Volk frei gewählten Status. ${ }^{13}$

Solange das besetzte Volk einem Gebietserwerb durch die Besatzungsmacht nicht zugestimmt hat, hat es dieses Selbstbestimmungsrecht noch nicht verwirkt. ${ }^{14}$ Das Recht auf Selbstbestimmung wird typischerweise durch ein Referendum ausgeübt. Doch wenn die Umstände keine Durchführung eines solchen Referendums, in welchem der freie Wille des Volkes zum Ausdruck gebracht werden könnte, erlauben, kann diese Aufgabe auch von traditionell als legitim anerkannten Repräsentanten, d.h. von Vertretern ethnischer, religiöser oder anderer verfassungsgebender Stämme und Gemeinschaften, wahrgenommen werden. ${ }^{15}$

Nachfolgend interessiert vor allem die letzte der vier genannten Optionen: Im englischen Originalwortlaut spricht die Friendly Relations Declaration von »the emergence into any other political status freely determined by a people«. Die Völkerrechtslehre schweigt sich zwar darüber aus, was konkret ein solcher anderer Status sein könnte, doch handelt es sich hierbei um ein klassisches Beispiel einer Auffangnorm: Jeder selbst gewählte politische Status ist grundsätzlich zulässig.

\subsection{Grenzen des Selbstbestimmungsrechts}

Das Völkerrecht - insbesondere das zwingende Völkerrecht (ius cogens) - selbst setzt jeder dieser vier Optionen jedoch gewisse Grenzen: Das Selbstbestimmungsrecht kann nur geltend gemacht werden, wenn die Sicherheitslage es aus Sicht der Menschenrechte und des humanitären Völkerrechts erlaubt, sowie soweit das Gewaltverbot und die Friedenssicherungspflicht gewahrt bleiben. Daraus ergeben sich Rechte und Pflichten für die jeweilige völkerrechtlich legitimierte Besatzungsmacht im Besonderen, aber auch für die Staatengemeinschaft im Allgemeinen: ${ }^{16}$ Es ist sowohl ihr Recht als auch ihre Pflicht, sich im Übergangsprozess für eine Lösung der Probleme einzuset-

13 Gusy, Christoph 1992: Selbstbestimmung im Wandel: Von der Selbstbestimmung durch den Staat zur Selbstbestimmung im Staat, in: Archiv für Völkerrecht 30, 399ff; Ermacora, Felix 2000, 288 und 290ff.

14 Gusy, Christoph 1992, 401f. So zum Beispiel im Fall des palästinensischen Volkes: Sutter, Patrick 2003b: Die Rolle der UNO und des Völkerrechts im Palästina-Konflikt: Ein Überblick, in: Jusletter, 2. Juni 2003, $29 \mathrm{f}$.

15 Vgl. SR-Resolution 1483 (2003), Ziff. 9: »Supports the formation, by the people of Iraq with the help of the Authority and working with the Special Representative, of an Iraqi interim administration as a transitional administration run by Iraqis, until an internationally recognized, representative government is established by the people of Iraq and assumes the responsibilities of the Authority.

16 Dies wird in der SR-Resolution 1483 (2003) sehr schön deutlich. Vergleiche auch den bereits erwähnten Report des High-level Panel, Ziff. 261: »There is a clear international obligation to assist States in developing their capacity to perform their sovereign functions effectively and responsibly.« zen, solange die erwähnten, durch völkerrechtlich zwingende Bestimmungen geschützten Güter in Gefahr sind. ${ }^{17}$ Jede Einschränkung des Selbstbestimmungsrechts, die diesem Zweck dient und sich auf das beschränkt, was völkerrechtlich notwendig ist, ist deshalb gerechtfertigt. Dies hat mit Paternalismus nichts zu tun, sondern ergibt sich aus der primären Sorge der VN-Charta um Frieden und Sicherheit auf der Welt. Es wäre vielmehr absurd, das Selbstbestimmungsrecht eines Volkes absolut zu verstehen und dadurch die Gewährleistung dieses Primärziels zu verunmöglichen. ${ }^{18}$ Folgende Analogie macht dies deutlich: In jedem demokratischen Rechtsstaat ist es zulässig, die Grundrechte von Individuen zum Schutz der öffentlichen Sicherheit einzuschränken, solange dies mit Blick auf die Verhältnismäßigkeit gerechtfertigt erscheint. Hier wie dort ist somit das Verhältnismäßigkeitsprinzip der gültige Maßstab.

Ist z.B. ein Überschwappen der unsicheren Lage in einem besetzten Staat auf die Nachbarstaaten zu befürchten, muss die Geltendmachung des Selbstbestimmungsrechts so lange warten, bis die Situation stabilisiert werden kann. Anders ist es natürlich, wenn der Grund für die Unruhen in der Besetzung selbst liegt. Dann muss aber mit genügender Gewissheit gewährleistet sein, dass mit einem Abzug der Besatzungstruppen und der Beendigung der Besetzung nicht ein Bürgerkrieg einsetzt. ${ }^{19}$

Die Pflichten beschränken sich allerdings nicht nur auf die militärische und polizeiliche Sicherheitsgewährleistung: Aus den genannten Gründen ist die Staatengemeinschaft bzw. die Besatzungsmacht auch berechtigt, sich in den Prozess der Verfassungsgebung einzumischen, was auch als Nation Building (oder State Building) bezeichnet wird. Die Bevölkerung muss zumindest mit Informationen darüber versorgt werden, welche Verfassungsoptionen ihr überhaupt zur Verfügung stehen, um Sicherheit und Frieden auch nach Beendigung der Besetzung gewährleisten zu können. ${ }^{20}$ Die Staatengemeinschaft darf und soll also beratende Funktionen wahrnehmen. Die Entscheidung über die Verfassung ist hingegen in jedem Fall alleiniges Recht des Volkes. Bei Beachtung dieser Kriterien ist das Nation Building ebenfalls eine Form der Intervention, die mit dem Selbstbestimmungsrecht kompatibel ist.

\subsection{Schlussfolgerungen für das Peacebuilding}

Nation Building - verstanden als »völkerrechtlich vermittelte Staatsbildung ${ }^{21}$ - ist somit im Lichte des Selbstbestimmungsrechts zulässig, solange die offerierten verfassungsrechtlichen

17 Williams, Ian 2002: Nur das letzte Mittel, Der Bericht der Axworthy-Kommission zur humanitären Intervention, in: Vereinte Nationen 1/2002, $10 \mathrm{ff}$.

18 Die in Ziff. 3 von Resolution 1514/XV zum Ausdruck kommende Ungeduld wird heute dafür verantwortlich gemacht, dass viele Kolonien komplett unvorbereitet in die Unabhängigkeit entlassen wurden, was sich noch lange Zeit später für ganze Weltgegenden destabilisierend auswirkte. Für Nachweise vergleiche Sutter, Patrick 2003a, 209f. und 212

19 Sutter, Patrick 2004: Völkerrecht und Truppenrückzug aus dem Irak: Die Rechte und Pflichten einer Besetzungsmacht, in: Neue Zürcher Zeitung, 31. März 2004, 9; Sutter, Patrick 2003a, $215 \mathrm{ff}$.

20 Dazu ist es unumgänglich, dass die Staatengemeinschaft die in früheren Fällen gemachten Erfahrungen aufarbeitet, um die Variablen zu identifizieren, von denen der Erfolg eines Verfassungstypus abhängt. Siehe etwa das integrative Konzept der Rechtsvergleichung bei Sutter, Patrick 2003a, $226 \mathrm{ff}$.

21 Ebenda, 205. 
Lösungen (a) die elementarsten Normen des Völkerrechts gewährleisten und (b) durch einen freien Beschluss des Staatsvolkes angenommen oder abgelehnt werden können, sei es in einem Referendum oder durch einen Beschluss ihrer traditionell legitimierten Repräsentanten. Das sind somit auch die Bedingungen, denen unter dem Gesichtspunkt des Selbstbestimmungsrechts entsprochen werden muss, wenn ein Staatsvolk oder das Volk eines werdenden Staates unter VN-Treuhandschaft gestellt werden soll.

Im Bereich des Peacebuilding ist jedoch praktisch per definitionem ausgeschlossen, dass eine Situation vorliegt, in welcher das Staatsvolk oder das Volk eines werdenden Staates auf der Basis des Selbstbestimmungsrechtes ihre Zustimmung zur Voraussetzung legitimen Handelns durch die Staatengemeinschaft machen können. Denn das Bestreben der Staatengemeinschaft besteht in diesen Fällen gerade darin, den Frieden erst noch zu sichern, weshalb das Selbstbestimmungsrecht unter Beachtung der Verhältnismäßigkeit sehr weitgehend eingeschränkt werden kann. Somit ist einzige Voraussetzung für die Unterstellung eines Staates unter VN-Treuhandschaft im Rahmen des Peacebuilding, dass diese Maßnahme zur Gewährleistung der elementarsten völkerrechtlichen Normen gemäß hiesigem Kapitel 2.2. geeignet und erforderlich ist und dass sich dieser Schritt auch in einer Abwägung mit dem Selbstbestimmungsrecht des Staatsvolks als zumutbar erweist.

Es sei allerdings nicht verschwiegen, dass die Unterscheidung zwischen Peacebuilding einerseits und Nation Building andererseits in jüngster Zeit an Klarheit eingebüßt hat. Dies ist vor allem Ausdruck einer aufgrund der ernüchternden Erfahrungen einsetzenden Neuorientierung im Bereich des Peacebuilding: Es setzt sich die Erkenntnis immer mehr durch, dass eine nachhaltige Befriedung nicht schon mit der Beseitigung des bewaffneten Konflikts erreicht ist, sondern dass dazu oft die gesamten Strukturen des Staates und der Gesellschaft reformiert werden müssen. Zudem schließt Peacebuilding vermehrt auch die Konfliktprävention ein - setzt somit also immer früher ein. ${ }^{22}$ Geht man davon aus, dass der Frieden erst gesichert ist, wenn eine stabile Verfassungsordnung geschaffen ist, die sich auch einige Jahre bewährt hat, und wirkt die Staatengemeinschaft im vorerwähnten Sinne am Prozess der Staatswerdung mit, so ist das Peacebuilding in der Tat weitgehend deckungsgleich mit dem Nation Building. Allerdings sind auch andere Konstellationen denkbar, weshalb die Unterscheidung der Begriffe nach wie vor sinnvoll ist.

Wir können für unsere Untersuchung den Schluss ziehen, dass es sowohl das Recht als auch die Pflicht der intervenierenden Staaten ist, sich im Übergangsprozess so lange für eine nachhaltige Friedenskonsolidierung einzusetzen, wie die erwähnten, durch völkerrechtlich zwingende Bestimmungen geschützten Güter in Gefahr sind. Die mit dem Peacebuilding einhergehenden Einschränkungen des Selbstbestimmungsrechts sind gerechtfertigt, wenn sie diesem Zweck dienen und sich auf das völkerrechtlich Notwendige beschränken.

22 Hänggi, Heiner 2005: Approaching Peacebuilding from a Security Governance Perspective, in: Alan Bryden, Heiner Hänggi (Hrsg.), Security Governance in Post-Conflict Peacebuilding, Münster, 10ff.

\section{Das Treuhandsystem der Vereinten Nationen}

Es stellt sich die Frage, ob das VN-Treuhandsystem selbst Beschränkungen kennt, die es verunmöglichen, dieses Institut als Mittel zum Peacebuilding zu verwenden. Die VN-Charta legt in ihren Kapiteln XII und XIII die Ziele und Voraussetzungen fest, unter welchen das VN-Treuhandsystem zur Anwendung gelangt. Dies betrifft »die Verwaltung und Beaufsichtigung der Hoheitsgebiete, die aufgrund späterer Einzelabkommen in dieses System einbezogen werden «. Diese Gebiete sind zu unterscheiden von denjenigen gemäß Kapitel XI, der »Erklärung über Hoheitsgebiete ohne Selbstregierung « (Artikel 73 und 74), welche für Hoheitsgebiete Anwendung findet, »deren Völker noch nicht die volle Selbstbestimmung erreicht haben $\ll$.

In beiden Fällen sollen die dort lebende Bevölkerung und das Wohlergehen der gesamten Staatengemeinschaft gefördert werden (Artikel 73, 74 und 76). ${ }^{23}$ Im Gegensatz zu Kapitel XI, das typischerweise Kolonien betrifft, bei denen noch nicht klar ist, wann sie der Selbstbestimmung teilhaftig werden, bezieht sich das VN-Treuhandsystem jedoch auf »Treuhandgebiete«, deren Bevölkerungen zur »Selbstregierung oder Unabhängigkeit« zu führen sind (Artikel 76 Bst. b). Im VNTreuhandsystem liegt die Verwirklichung dieser Ziele bei der durch völkerrechtlichen Organisationsakt bestellten Administrationsbehörde. Das sog. Treuhandabkommen (Artikel 79), das vom Sicherheitsrat genehmigt werden muss (Artikel 83), enthält die Bestimmungen, nach denen das Treuhandgebiet zu verwalten ist (Artikel 81). Das Treuhandmandat kann gemäß Artikel 81 einem Staat, einer Staatengruppe oder den VN selbst übertragen werden. Nachfolgend gehen wir von der dritten Option aus.

Dem System liegt das Mandatssystem des Völkerbundes zugrunde. Deshalb sieht Artikel 77 Absatz 1 als ersten Anwendungsbereich "gegenwärtig bestehende Mandatsgebiete« vor (Bst. a). Sodann kommen »Hoheitsgebiete, die infolge des Zweiten Weltkriegs von Feindstaaten abgetrennt wurden « in Frage (Bst. b). Und schließlich können Hoheitsgebiete »von den für ihre Verwaltung verantwortlichen Staaten freiwillig in das System einbezogen werden « (Bst. c). Ganz im Sinne des Grundsatzes der souveränen Gleichheit aller Mitglieder der Vereinten Nationen (Artikel 2 Absatz 1) schließt Artikel 78 »Hoheitsgebiete, die Mitglied der Vereinten Nationen geworden sind « vom Anwendungsbereich des VN-Treuhandsystems aus. Die Ziele, die gemäß dem bereits erwähnten Artikel 76 mit dem Treuhandsystem erreicht werden sollen, lauten:

- die Festigung des Weltfriedens und der internationalen Sicherheit,

- die Förderung des politischen, wirtschaftlichen, sozialen und erzieherischen Fortschritts der Einwohner der Treuhandgebiete,

23 Zur Geschichte dieses Grundgedankens siehe Lakshmi-Narayan, Chetlur Venkatramana 1995: Analysis of the principles and system of international trusteeship in the Charter: A study of the origin, principles and application in international law, Geneva, 11ff.; Rauschning, Dietrich 2000: United Nations Trusteeship System, in: Rudolf Bernhardt (Hrsg.): Encyclopedia of Public International Law, volume IV, 1193. 
- die Achtung der Menschenrechte und Grundfreiheiten und

- die Gleichbehandlung aller Mitglieder der VN und ihrer Staatsangehörigen.

Diese Zielsetzungen lassen das VN-Treuhandsystem als geradezu ideal erscheinen zur Einschränkung des Selbstbestimmungsrechts: Denn, wie Kapitel 2 gezeigt hat, ist eine Einschränkung genau zum Zwecke der Gewährleistung dieser grundlegenden Normen zulässig. Man möchte einzig ergänzend hinzufügen, dass diese Lösung nur zu rechtfertigen ist, wenn es nach dem Verhältnismäßigkeitsprinzip keine andere, das Selbstbestimmungsrecht weniger einschränkende Maßnahme gibt, um diese grundlegenden Normen gewährleisten zu können.

Wenn diese Voraussetzungen erfüllt sind, ist die Zustimmung des betroffenen Staatsvolkes nicht notwendig. Hätten die alliierten Kräfte nach der Legitimation ihrer Besetzung durch SR-Resolution 1483 (2003) den Irak also dem VN-Treuhandsystem übergeben können, solange sie noch Besatzungsmacht waren? Dazu sind zwei bereits erwähnte Normen in ihrer Bedeutung genauer zu beleuchten: Artikel 77 Absatz 1 Bst. c und Artikel 78.

Bei Artikel 77 Absatz 1 Bst. c dachte man an diejenigen Hoheitsgebiete ohne Selbstregierung gemäß Kapitel XI, die weder Mandatsgebiet aus der Völkerbundszeit ${ }^{24}$ noch im Zweiten Weltkrieg abgetrennte Hoheitsgebiete ${ }^{25}$ waren. Bis zum heutigen Tag gibt es jedoch keinen einzigen Anwendungsfall von Artikel 77 Absatz 1 Bst. c. Es spricht allerdings nichts dagegen, diese Bestimmung generell auf Gebiete anzuwenden, die nach einer völkerrechtlich legitimen Intervention besetzt sind bzw. deren Besetzung nachträglich durch eine Resolution des Sicherheitsrates legitimiert wurde. Solange der Irak also nach der Intervention der alliierten Kräfte ein Hoheitsgebiet ohne Selbstregierung war, weil das irakische Volk die Selbstregierung verloren und noch nicht vollumfänglich wiedererlangt hatte, und nachdem diese Besetzung durch SR-Resolution 1483 (2003) legitimiert wurde, hätten die alliierten Kräfte als Besatzungsmächte den Irak somit freiwillig dem VN-Treuhandsystem unterstellen können. Allerdings steht ein Vorgehen nach Artikel 77 Absatz 1 Bst. c unter dem Vorbehalt, dass es mit Artikel 78 konform sein muss. Da der Irak auch während und nach der Invasion jederzeit Mitglied der Vereinten Nationen war, scheint hier eine VN-Treuhandschaft ausgeschlossen. Was ist die ratio legis dieser Bestimmung?

Artikel 78 wurde eingeführt, um Befürchtungen von Syrien und Libanon zu zerstreuen: Diese hatten als frühere Mandatsgebiete Frankreichs 1944/45 keine überleitenden Verträge mit Frankreich geschlossen. Ihre Kriegserklärung im Februar 1945 gegenüber Deutschland berechtigte die beiden Staaten aber zur Teilnahme an der VN-Gründungskonferenz. Sie wollten mit der Bestimmung des Artikels 78 sicherstellen, dass sie als VN-Gründungsmitglieder nun nicht mehr unter Treuhand-

24 Wie Kamerun, Togo, Transjordanien und so weiter. Vergleiche dazu die Übersicht von Lakshmi-Narayan, Chetlur Venkatramana 1995, 86ff; Rauschning, Dietrich 1991: Artikel 77, in: Bruno Simma (Hrsg.): Charta der Vereinten Nationen, Kommentar, $1195 \mathrm{f}$.

25 Wie Somaliland. Siehe Lakshmi-Narayan, Chetlur Venkatramana 1995, 103 ff.; Rauschning, Dietrich 2000, 1196. schaft gestellt werden konnten. ${ }^{26}$ Es ist deshalb fraglich, ob man aus Artikel 78 generell schließen darf, dass das Volk eines VN-Mitgliedstaates, das temporär nicht in der Lage ist, sich selbst zu regieren, nicht dem $\mathrm{VN}$-Treuhandsystem unterstellt werden darf.

Meines Erachtens ist dies aufgrund folgender Überlegungen zu verneinen: Einmal abgesehen von den Vetomächten im Sicherheitsrat ist kein VN-Mitgliedstaat per se von der Möglichkeit ausgenommen, nach einer militärischen Intervention gemäß Kapitel VII der VN-Charta ein besetztes Gebiet zu sein. Die völkerrechtlich legitime Besatzungsmacht ist in diesem Fall ermächtigt, die Besetzung so lange aufrechtzuerhalten, wie es nach Maßgabe des Selbstbestimmungsrechts verhältnismäßig ist, um die Menschenrechte und den Frieden zu sichern. Da auch die Vereinten Nationen die Treuhandschaft nur so lange aufrechterhalten dürfen, wie dies vor dem Selbstbestimmungsrecht zulässig ist, macht eine Unterstellung unter die treuhänderische Verwaltung der Vereinten Nationen für das besetzte bzw. verwaltete Staatsvolk keinen Unterschied in Bezug auf sein Selbstbestimmungsrecht. Nur wenn man nicht (an)erkennt, dass die Frage der Zulässigkeit einer VNTreuhandschaft nach Maßgabe des Selbstbestimmungsrechts zu beurteilen ist, kann man sich dieser Lösung nicht anschließen. Sollten sich die VN-Mitgliedstaaten entschließen, vom Treuhandsystem für das Peacebuilding Gebrauch zu machen, wäre aber um der Klarheit willen eine Streichung von Artikel 78 sicherlich sinnvoll.

Die Konstellation im Kosovo ist insofern speziell, als nicht der gesamte jugoslawische Staat von der NATO besetzt wurde, sondern nur ein Teilgebiet. Alle nachfolgenden Ausführungen gehen von der Annahme aus, dass die NATO-Intervention rechtmäßig erfolgte, ohne damit tatsächlich etwas über die zweifelhafte Völkerrechtskonformität sagen zu wollen. Die Resolution 1244 des VN-Sicherheitsrates betont, dass das Kosovo als integraler Bestandteil des jugoslawischen Staates angesehen wird. ${ }^{27}$ Deshalb stünde bei einer Verwaltung des Kosovos durch das VN-Treuhandsystem die territoriale Integrität Jugoslawiens in Widerspruch zum Ziel gemäß Artikel 76 Bst. b, das Volk auf die »Selbständigkeit oder Unabhängigkeit « vorzubereiten. Man kann diese beiden Ziele immerhin insoweit vereinen, als dass man mit dem Ziel der Selbständigkeit oder Unabhängigkeit auch eine Autonomie ohne Sezession verstehen kann. In diesem Fall steht der Anwendung der vorstehend beschriebenen, vom Selbstbestimmungsrecht her argumentierenden Lösung auch in einer solchen Konstellation nichts im Wege. Ich bin deshalb der Ansicht, dass die NATO nach einer völkerrechtskonformen Intervention im Kosovo bzw. nach der völkerrechtlichen Legitimation ihrer Besetzung durch den VN-Sicherheitsrat berechtigt gewesen wäre, dieses Teilgebiet der VN-Treuhandschaft zu übergeben. ${ }^{28}$ Andernfalls

26 Lakshmi-Narayan, Chetlur Venkatramana 1995, 86; Rauschning, Dietrich 1991: Artikel 78, in: Kommentar, Bruno Simma (Hrsg.): Charta der Vereinten Nationen, $1195 \mathrm{f}$

27 Auf die anstehenden Verhandlungen zur Frage des künftigen Status des Kosovo gehen wir hier nicht ein.

28 Aufgrund dieser Überlegungen unterscheidet sich meine Einschätzung von derjenigen von Zimmermann, Andreas/Stahn, Carsten 2002: Yugoslav Territory, United Nations Trusteeship or Sovereign State? Reflections on the Current and Future Legal Status of Kosovo, in: Nordic Journal of International Law 70,436 . 
wäre zu prüfen, ob nicht die VN-Charta angepasst werden sollte, um den Bedürfnissen in einer speziellen Konstellation wie im Kosovo gerecht zu werden.

Wie sieht es aus, wenn ein Staatsvolk in Ausübung seines Selbstbestimmungsrechts im Rahmen der vierten Option gemäß der Friendly Relations Declaration beschließt, sich selbst für eine Übergangsphase dem VN-Treuhandsystem zu unterstellen? Könnte sich also z.B. das irakische Volk (bzw. die in Abschnitt 2.1 erwähnten, traditionell als legitim anerkannten Repräsentanten des Volkes) selbst freiwillig dem VN-Treuhandsystem unterstellen? Es ist m.a.W. zu klären, ob Artikel 77 Absatz 1 Bst. c die freiwillige Unterstellung eines Staates durch eigenen Beschluss zulässt oder ob das VN-Treuhandsystem nach dem Willen der VN-Charta dafür keine Anwendung finden soll. Die Analyse der Vorarbeiten zeigt, dass an diesen Fall schlicht nicht gedacht wurde. ${ }^{29}$ Man kann deshalb nicht sagen, dass die VN-Gründungskonferenz solche Fälle absichtlich nicht durch das Treuhandsystem verwaltet haben wollte. Ein solches Szenario schien, wie bereits erwähnt, in einer Zeit, da alle Staatsvölker bzw. Völker werdender Staaten nach Unabhängigkeit strebten, einfach absolut undenkbar. Es gestaltet sich deshalb als einigermaßen schwierig, in dieser Frage zu einer zufrieden stellenden Interpretation zu gelangen.

Eine vertretbare Lösung könnte anhand folgender Leitlinien gefunden werden: Würde man es einem Staatsvolk verwehren, sich selbst freiwillig dem VN-Treuhandsystem zu unterstellen, würde man es ihm verwehren, die Ziele von Artikel 76 unter dem Schutzmantel der treuhänderischen Verwaltung verwirklichen zu wollen. Es bestehen keine Hinweise dafür, dass die Autoren der VN-Charta eine solche Diskriminierung gewollt haben bzw. dass sie, hätten sie diesen Fall bedacht, ihn explizit ausgeschlossen hätten. Eine solche Ungleichbehandlung wäre mit sachlichen Gründen kaum zu rechtfertigen und würde den wichtigsten Zielen der VN-Charta entgegenstehen.

Da sich also aus Artikel 77 Abs. 1 lit. c keine Einwände ergeben, bleibt noch die Vereinbarkeit mit Artikel 78 zu klären: Diese Bestimmung kann auf keinen Fall so verstanden werden, dass er es einem VN-Mitglied verbieten möchte, sich selbst für einen gewissen Zeitraum unter VN-Treuhandschaft zu stellen. Es schien der Gründungskonferenz auch hier als schlicht undenkbar, dass ein souveräner VN-Mitgliedstaat jemals in eine Situation kommen könnte, in der er seinen fundamentalen Interessen am besten dienen zu können glaubt, indem er sich unter diesen Schutzmantel begibt.

Von den Schwierigkeiten überhaupt nicht betroffen ist der Fall, dass sich ein Staat entschließt, ein Teilgebiet, über das er die Kontrolle zu verlieren droht, der VN-Treuhandschaft zu unterstellen. Zu denken wäre etwa an den Fall, dass Russland Tschetschenien oder der Sudan Darfur der treuhänderischen Verwaltung der Vereinten Nationen überlässt. Dies könnte eine entspannende Wirkung auf den Konflikt haben und Zeit und Raum schaffen, um - entlastet von den Wirren eines Bürgerkriegs - nach Lösungen zu suchen. In einer solchen Kon-

29 Vergleiche schon Section B paragraph 3 des Working Paper an der UN-Gründungskonferenz - abgedruckt bei Lakshmi-Narayan, Chetlur Venkatramana 1995, 37 - sowie dessen weitere Evolution bis zur definitiven Fassung von Kapitel XII und XIII der VN-Charta. Zu Letzterem siehe ebenda, 47ff. stellation erachte ich eine VN-Treuhandschaft ohne Zweifel als eines der wirkungsvollsten Mittel. Da die meisten Staaten aber befürchten würden, dass eine solche freiwillige Unterstellung eines Teilgebietes als Zeichen der Schwäche gedeutet oder dass sie eine Sezession dieses Gebietes präjudizieren würde, ist nicht davon auszugehen, dass sie von dieser an sich klugen Lösung Gebrauch machen werden. Die Befürchtung eines Präjudizes ist insofern berechtigt, als das Treuhandsystem gemäß Artikel 76 Bst. b das Staatsvolk »zur Selbständigkeit oder Unabhängigkeit « führen soll. Für entsprechende Auswege sei auf die vorstehenden Ausführungen in Bezug auf das Kosovo verwiesen. So würden sich Staaten dereinst eventuell einmal entscheiden, mit den Vereinten Nationen über ein Treuhandschaftsabkommen zu verhandeln, das für die Stabilisierung und die Befriedung des Teilgebietes unter Beachtung der zwingenden Normen des Völkerrechts sorgen könnte.

\section{Abschließende Bemerkungen}

Die Vereinten Nationen zogen in ihrer bisherigen Geschichte einen klaren Trennstrich zwischen den Missionen nach Kapitel VI und VII einerseits und der Verwaltung von Gebieten nach Kapitel XI bis XIII andererseits. Da sich jedoch die Ziele, die mit den Kapiteln XI bis XIII angestrebt werden, mit denjenigen decken, die insbesondere durch Interventionen nach Kapitel VII verwirklicht werden sollen, erscheint diese Trennung künstlich.

Kapitel XI bis XIII sind für Gebiete, die sich in einer Übergangsphase befinden, geschaffen worden, um diese an die elementaren Standards der Vereinten Nationen heranzuführen. Die Mandatsmacht erhält einen klaren Auftrag und soll diesen im Rahmen einer längerfristigen Tätigkeit erfüllen.

Das Problem bei vielen der bisherigen Missionen nach Kapitel VII scheint mir hingegen darin zu bestehen, dass der längerfristige Verbleib in einem Gebiet unter diesem Titel der VNCharta nicht explizit vorgesehen ist. Zwar bestreitet niemand, dass der Verbleib zur Stabilisierung zulässig, ja geboten ist. Es ist jedoch unklar, welche Kompetenzen einer internationalen Administrationsbehörde von Kapitel VII gedeckt sind. ${ }^{30}$ Als Folge dieses Handelns im freien Raum wurde jedes Mandat der jeweiligen internationalen Verwaltung neu ausgestaltet - mit unterschiedlichen Kompetenzen und unterschiedlichen Zielen, ohne dass diese Unterschiede immer begründbar waren. Darunter leidet die Rechtssicherheit in der Staatenpraxis. Insgesamt ist das bisherige Vorgehen unsystematisch und völkerrechtlich problematisch. ${ }^{31}$

Wie die Vereinten Nationen mit der Schaffung der PBC anerkennen, besteht bezüglich Peacebuilding Handlungsbedarf. Allerdings haben sie sich entschieden, dem bisherigen Grundmodell treu zu bleiben. Neu wird die PBC als zentrale Instanz funktionieren, in der das Wissen und die Erfahrungen gesammelt und abgefragt werden können sowie die Bestrebungen

30 Zimmermann, Andreas/Stahn, Carsten 2002: 437ff.

31 Vergleiche die Übersicht bei Zimmermann, Andreas/Stahn, Carsten 2002: 429ff; Caplan, Richard 2002: A New Trusteeship? The International Administration of War-torn Territories, Oxford, 13ff.; Chopra, Jarat 1995: UN Civil Governance-in-Trust, in: Thomas G. Weiss (Hrsg.): The United Nations and Civil Wars, Boulder/London, 69ff. 
der verschiedenen Akteure koordiniert werden. Damit bleibt meines Erachtens aber ein wichtiges Problem ungeklärt: In welchem Rahmen sollen die Peacebuilding-Missionen zukünftig stattfinden?

Aufgrund des vorstehend Ausgeführten scheint mir das VN-Treuhandsystem in denjenigen Fällen sehr geeignet, in welchen es darum geht, den Konflikt zwischen Parteien abzukühlen, die im Moment aufgrund des mangelnden Vertrauens noch gar nicht in der Lage sind, sich sogleich über die Ausgestaltung der künftigen Ordnung zu einigen. Es geht also vor allem um Konflikte, von denen weite Teile der Zivilbevölkerung betroffen sind und in welchen schwerwiegende Verbrechen begangen wurden. Die treuhänderische Verwaltung wird konsequent auf die Verwirklichung der Ziele der VN-Charta ausgerichtet. Sobald sich die Einschränkungen des Selbstbestimmungsrechts nicht mehr rechtfertigen lassen, was bedeutet, dass der notstandsähnliche Zustand beendet werden konnte, ist die Treuhandschaft aufzuheben.

Grund dafür, dass diese Option bisher kaum in Erwägung gezogen wurde, ist vermutlich der paternalistische Geruch, der dem VN-Treuhandsystem anhaftet. Wie jedoch vorstehend gezeigt werden sollte, beruht dies bei konsequenter Orientierung am Selbstbestimmungsrecht und dessen zulässigen Einschränkungen auf einem Missverständnis. Gefährdungen der internationalen Sicherheit und des internationalen Friedens sowie anderer Normen des zwingenden Völkerrechts vermögen verhältnismäßige Beschränkungen zu rechtfertigen, wie am Beispiel einer militärischen Intervention nach Kapitel VII deutlich wird.

Meines Erachtens präjudiziert die Errichtung der PBC in Bezug auf den hier vorgestellten Vorschlag nichts. Das VNTreuhandsystem ist ein Gefäß, das für die Anwendung auf den konkreten Fall zwar den notwendigen Raum lässt, aber dennoch einen stets gleich bleibenden Rahmen vorgibt. Es fügt sich in die Systematik der VN-Charta nahtlos ein, auch wenn man es vom Ziel der Dekolonisierung löst. Dass der Treuhandrat - mangels kolonisierter Gebiete - seit 1994 nicht mehr aktiv ist, heißt nicht, dass das Treuhandsystem an sich nutzlos geworden ist. Für einzelne spezielle Konstellationen empfehlen sich jedoch Anpassungen der VN-Charta. Da im Moment Revisionen der VN-Charta als chancenlos erachtet werden, ist zu befürchten, dass die trotz $\mathrm{PBC}$ verbleibende Lücke für den Moment offen bleibt.

\section{Millionenfach gelesen}

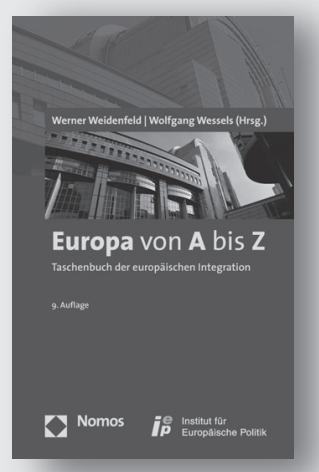

\section{Europa von A bis Z}

Taschenbuch der europäischen Integration

Herausgegeben von Prof. Dr. Dr. h.c. Werner Weidenfeld, Centrum für angewandte Politikforschung und Prof. Dr. Wolfgang Wessels, Jean Monnet Lehrstuhl an der Universität Köln

9. Auflage 2006, 496 S., brosch., 19,90 €, ISBN 3-8329-1378-5

In Europa überschlagen sich die Ereignisse. Da ist es wichtig, den Überblick zu behalten. Die Neuauflage des Taschenbuches der europäischen Integration hilft dabei durch

- ein umfangreiches Lexikon mit den wichtigsten Themen und Begriffen zu Europa,

- Statistiken und Übersichten zur Bevölkerung, Wirtschaft und Politik,

- eine informative Einführung in die Geschichte der Europäischen Integration und

- eine umfangreiche Linksammlung zu Europa.

Renommierte Autorinnen und Autoren erläutern kurz und verständlich die Kernthemen Europas. Das Taschenbuch liefert somit zentrales Hintergrundwissen und ist der perfekte Wegweiser durch das aktuelle Europa: ein unentbehrliches Nachschlagewerk. 